\title{
Early postoperative discharge following radical vulvectomy and bilateral inguinal lymphadenectomy for vulvar carcinoma
}

\author{
Nikolaos Burbos • Mazen Abu-Freij • \\ Mercedes Perez-Morales • Joaquin J. Nieto
}

Received: 5 April 2009 /Accepted: 19 June 2009 / Published online: 18 July 2009

(C) Springer-Verlag 2009

\begin{abstract}
Currently, no robust evidence exists for the optimal period for maintaining the suction drainage in the groin incisions for women who undergo inguinal lymphadenectomy for vulvar carcinoma. In many cases, this may take more than 2 weeks. Some authorities advocate early drain removal at $72 \mathrm{~h}$ after surgery, but this approach is associated with increased risk of lymphocyst formation. We attempted to discharge women with suction drains in situ within 48 to $72 \mathrm{~h}$ following the surgery. Four patients that underwent vulvectomy and bilateral inguinal lymphadenectomy for vulval cancer were discharged home within 48 to $72 \mathrm{~h}$ postoperatively with suction drains in situ. The mean age was 61.5 years. The average number of groin lymph nodes removed was 9.12 per patient. Only one patient had nodes positive for disease (bilaterally). Drains were removed within 7 to 10 days following the discharge. Wound healing was satisfactory in all cases. No cases of wound breakdown were recorded. There was only one case of a small lymphocyst $(<3 \mathrm{~cm})$ that resolved spontaneously within 8 weeks. Early postoperative discharge with suction
\end{abstract}

\footnotetext{
N. Burbos Ipswich, UK

M. Abu-Freij $\cdot$ M. Perez-Morales $\cdot$ J. J. Nieto

Department of Obstetrics and Gynecology,

Norfolk and Norwich University Hospital,

Norwich, UK

N. Burbos $(\square)$

60 Old Street, Haughley,

IP14 3NX Stowmarket, UK

e-mail: nikosburbos@hotmail.com
}

Department of Obstetrics and Gynecology, Ipswich Hospital, drains in situ appears safe for women that undergo inguinal lymphadenectomy for vulvar carcinoma.

Keywords Vulvar Carcinoma - Inguinal . Lymphadenectomy $\cdot$ Discharge

\section{Introduction}

Vulvar cancer is the fourth most common gynaecological cancer, following endometrial, ovarian and cervical cancer. Surgical treatment of vulvar cancer should be individualised so as to perform the most conservative operation that is consistent with the cure of the disease.

The technique of inguinofemoral node dissection through separate incisions (three incision technique) significantly decreases postoperative hospital stay and the incidence of chronic lymphoedema [1]. This technique requires suction drainage to be placed in each groin incision at the end of the procedure to prevent lymph collection and to reduce the incidence of lymphocyst formation and wound breakdown.

Currently, no robust evidence exists for the optimal period for maintaining the suction drainage. In many cases, this may take more than 2 weeks. Some authorities advocate early drain removal at $72 \mathrm{~h}$ after surgery, but this approach is associated with increased risk of lymphocyst formation. Others recommend removal once drainage falls below a specific threshold (usually $30 \mathrm{ml}$ over $24 \mathrm{~h}$ ).

In an attempt to decrease the postoperative risks associated with prolonged hospital stay (e.g. venous thromboembolism, hospital-acquired infections), we discharged women that underwent radical vulvectomy and bilateral inguinal lymphadenectomy with suction drains in situ within 48 to $72 \mathrm{~h}$ postoperatively. 


\section{Summary of the cases}

Over a 4-month period, four women treated for vulvar carcinoma underwent radical vulvectomy and bilateral inguinal lymphadenectomy at a referral centre for gynaecological oncology. The mean age of the patients was 61.5 years (range $44-82$ years) and their mean body weight $87.1 \mathrm{~kg}$ (range $64-103.5 \mathrm{~kg}$ ). Three patients did not have any significant medical history on admission. One patient suffered from cardiomyopathy and sarcoidosis.

The procedure performed was radical vulvectomy and bilateral inguinal lymphadenectomy. The inguinal lymph nodes were dissected to below the inguinal ligament and careful attention to haemostasis was given. Drainage using a closed suction device was placed in situ and the wound was closed in layers.

In order to reduce the risk of thromboembolic disease, early ambulation was encouraged. All patients received prophylactic low-molecular-weight heparin during their hospital stay and graduated compression elastic stockings were fitted. No cases of venous thromboembolism were reported. Intravenous antibiotics were given preoperatively.

Indwelling catheter was removed $48 \mathrm{~h}$ following the operation. One patient developed urinary retention following the removal of the catheter and needed recatheterisation. Three patients were discharged home on the second postoperative day and the later patient on the third day.

Patients were discharged home with suction drains in situ. Advice about meticulous perineal hygiene was given. This was supported with the use of information leaflets. Regarding the vulvar wound, sitz baths were recommended. Perineum was kept dry following the baths with the use of a hair dryer. We did not recommend wound dressing for the vulvar and inguinal wounds. A supply of oral analgesics and laxatives were provided on discharge from the hospital for use when required. Carrying bags were given to facilitate the handling of the inguinal drains and improve patient's mobilisation. Clear instructions were given to them at discharge regarding the handling of the suction drains. Instructions to contact hospital if necessary were given. The community nurses were asked to review the drains and empty the bottles if necessary. The nurses visited the patients regularly and reported no difficulties during this period in managing the drains.

The patients returned to clinic within 7 to 10 days following the discharge. Wound sutures and drains were removed at that time. Healing of the wounds was satisfactory in all cases. One patient received a course of Metronidazole for a week as a swab taken from the groin wound showed growth of mixed anaerobes of doubtful clinical significance, but there were no clinical signs of infection. Only one patient developed a small lymphocyst (less than $3 \mathrm{~cm}$ in diameter) that was noted during the first postoperative visit in the clinic and resolved with conservative management within 8 weeks. No other postoperative complications were reported.

Patients' satisfaction was high with the early postoperative discharge and no concerns were expressed.

Histological examination of the specimens showed squamous cell carcinoma in all of the cases. Lymph nodes were negative for disease in three cases. In one patient, disease was present in inguinal lymph nodes bilaterally.

\section{Discussion}

Vulvar carcinoma is uncommon, representing approximately $5 \%$ of malignancies of the female genital tract. It occurs most frequently in women between the ages of 65 and 75, but incidence appears to be increasing among young women [2]. Although there is no standard approach applicable to every patient with vulvar carcinoma, surgical management is the cornerstone of treatment, comprising resection of the primary tumour and bilateral inguinal lymphadenectomy.

Postoperative complications following surgery for vulvar carcinoma have significant impact on long-term psychosexual and physical well-being. The most common complication after groin dissection are wound infection, wound breakdown, lymphoedema and lymphocyst formation. In one series, these complications were reported in $66 \%$ of the patients [3]. Urinary tract infection, thromboembolism and osteitis pubis are additional potential complications. One major late complication is chronic lymphoedema, which occurs in approximately $30 \%$ of patients [4]. The technique of inguinofemoral node dissection through separate incisions significantly decreases postoperative hospital stay and the incidence of chronic lymphoedema [1], although there is still significant risk of wound breakdown and lymphocyst formation.

In England, for patients that undergo inguinal lymphadenectomy for metastatic disease from penile, vulval, anal and cutaneous malignancies, the mean inpatient stay is 12.8 days [5]. There are theoretical advantages of discharging patients home earlier with suction drains in situ; this approach encourages early ambulation (thereby reducing thromboembolism risk) and reduces the risk of hospitalacquired infections. Also, maintaining the suction drains in situ does not increase the risk of lymphocyst formation and lymphoedema. In our series, early postoperative discharge within 48 to $72 \mathrm{~h}$ following surgery appears to be safe and without any major risks to the patient. No cases of wound breakdown were reported. Clinically, there was no evidence of wound infection in all patients. The small lymphocyst that was noted in one of the patients resolved spontaneously.

In order to achieve optimal results, an integrated care pathway is required. This should involve a multidisciplin- 
ary team approach including hospital specialists, oncology specialist nurses, community nurses and general practitioners. Community nurses are able to develop a close therapeutic relationship with patients and their families with enhanced knowledge of the patients' condition that enables them to offer a high standard of care and support. Clear protocols for contact and referral to hospital are in place if required.

Until new methods such as sentinel node dissection, which may permit the selective use of complete node dissection, become widely available, we recommend early postoperative discharge with suction drains in situ for women undergoing bilateral inguinal lymphadenectomy as part of surgery for vulvar carcinoma.

\section{References}

1. Hacker NF, Leuchter RS, Berek JS, Castaldo TW, Lagasse LD (1981) Radical vulvectomy and bilateral inguinal lymphadenectomy through separate groin incisions. Obstet Gynecol 58(5):574-579

2. Jemal A, Tiwani RC, Murray T, Ghafoor A, Samuels A, Ward E et al (2004) Cancer statistics 2004. CA Cancer J Clin 54(1):8-29

3. Gaarenstroom KN, Kenter GG, Trimbos JB, Agous I, Amant F, Peters AA, Vergote I (2003) Postoperative complications after vulvectomy and inguinofemoral lymphadenectomy using separate groin incisions. Int J Gynecol Cancer 13(4):522-527

4. Gould N, Kamelle S, Tillmanns T, Scribner D, Gold M, Walker J et al (2001) Predictors of complications after inguinal lymphadenectomy. Gynecol Oncol 82:329-332

5. Swan MC, Furniss D, Cassell OC (2004) Surgical management of metastatic inguinal lymphadenopathy. BMJ 329(7477):1272-1276 